\title{
20 Decision Fusion in Cognitive Wireless Sensor Networks
}

\author{
Andrea Abrardo, Marco Martalò, and Gianluigi Ferrari
}

\section{CONTENTS}

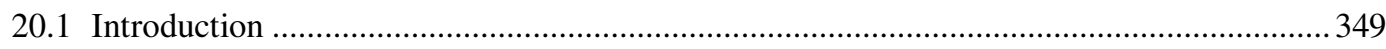

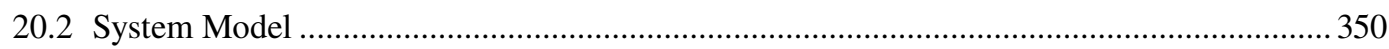

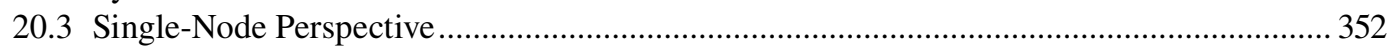

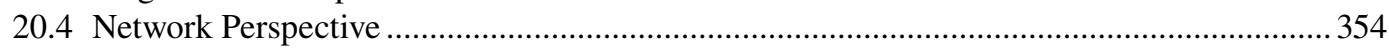

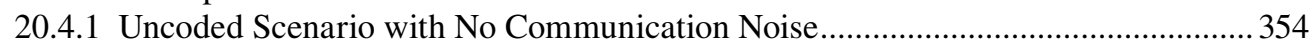

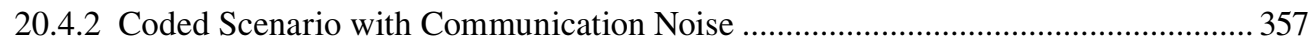

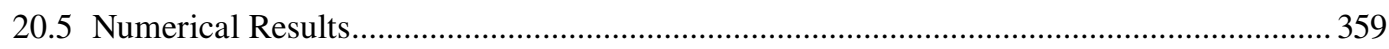

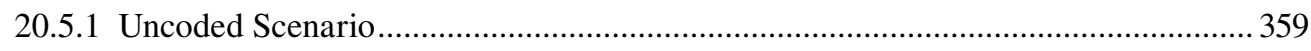

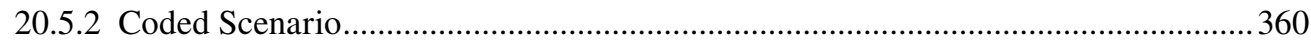

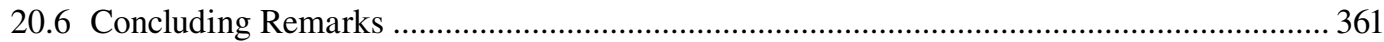

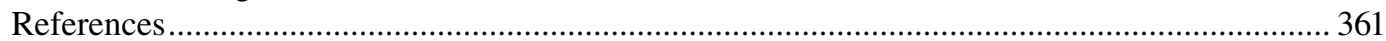

\subsection{INTRODUCTION}

With the development of wireless communications in the last few years, most of the available spectrum has been fully allocated. On the other hand, recent investigations on the actual spectrum utilization have shown that a portion of the licensed spectrum is largely underutilized [1]. As a matter of fact, the so-called spectrum scarcity problem is due mostly to an inefficient spectrum allocation policy rather than to actual physical spectrum shortage. Accordingly, to "chase" the explosion of wireless communications, novel solutions should be envisaged.

Dynamic spectrum access (DSA) has been considered to achieve a more efficient radio spectrum utilization [2,3]. In DSA, part of the spectrum can be allocated to one or more users, which are called primary users (PUs). Such a spectrum is not exclusively dedicated to PUs, although they have higher priority than other users, which are referred to as secondary users (SUs). In particular, SUs can access the same spectrum as long as the PUs are not temporally using it or can share the spectrum with the PUs as long as the PUs can be properly protected. By doing so, the radio spectrum can be reused in an opportunistic manner or shared all the time; thus the spectrum utilization efficiency can be improved significantly.

To support DSA, SUs are required to capture or sense the radio environment, and an SU with such a capability is also called a cognitive radio (CR) [4,5]. One of the main tasks of CR is represented by spectrum sensing (SS), defined as the task of finding spectrum holes [6], that is, portions of the spectrum allocated (licensed) to some primary users but left unused for a certain time. On the other hand, SS from a single node does not always guarantee satisfactory performance because of noise uncertainty, the intrinsic random nature of the nodes' positions, and unpredictable channel fluctuations. For example, a CR user cannot detect the signal from a primary transmitter behind a high building and may decide to access the licensed channel, thus interfering with the primary receiver.

On the other hand, collaboration of multiple users in SS may highly improve SS performance by introducing a form of spatial diversity $[7,8]$. In cooperative SS, CR users first send the collected 
data to a combining user or fusion center (FC). Alternatively, each user may independently perform local decisions and then report binary decisions to the FC. Finally, the FC takes a decision on the presence or absence of the licensed signal based on the received information.

In this chapter, we focus on a cognitive WSN, where a primary wireless sensor network (PWSN) is co-located with a cognitive (or secondary) sensor network (CWSN). In particular, the nodes of the CWSN reach their associated access point (AP) directly (single hop). The frequency spectrum that is shared by PWSN and CWSN is divided into subchannels, which can be assigned by the PWSN, whereas the nodes of the CWSN cooperate to sense the frequency spectrum and estimate the free subchannels that can be used to transmit their data. The secondary nodes transmit the packets containing the observations on the channels' statuses to their FC, embedded in the secondary AP, which makes a final decision about the status (free or busy) of each subchannel and broadcasts this information to all secondary nodes. The correlation, in the sensing operation, among the secondary nodes is taken into account. In particular, we provide a simple analytical model to characterize the local sensing performance per subchannel, in terms of probabilities of missed detection (MD) and false alarm (FA). Moreover, we propose a joint source channel coding (JSCC) scheme, in the CWSN, to exploit the source correlation to improve the reliability of the final decision taken by the secondary FC. By relying on recent results on the design of practical decoding and fusion rules for multiple access schemes with correlated sources [9], we derive an effective fusion rule and an associated iterative decoding strategy for joint channel decoding (JCD) at the secondary FC.

The rest of the chapter is structured as follows. In Section 20.2, we present the reference scenario. In Section 20.3, we analyze the performance from a single node perspective. Then, in Section 20.4 we analyze the performance from a network point of view, distinguishing between uncoded and coded scenarios. In Section 20.5, both theoretical and simulation results are provided. Finally, concluding remarks are given in Section 20.6.

\subsection{SYSTEM MODEL}

The scenario of interest is shown in Figure 20.1. The FC is placed at the center of the cell, while secondary and primary nodes are independent and identically distributed (i.i.d.) according to a uniform distribution in a circular cell with a given radius $R$. A logical description of the scenario is given in Figure 20.2. Let us denote as cognitive user equipment (CUE) and primary user equipment (PUE) the secondary and primary nodes, respectively** The number of PUEs and CUEs is equal to $P$ and $N$, respectively. Each PUE is assigned one channel among a set of $N_{\mathrm{ch}}$ orthogonal subchannels, for example, a set of nonoverlapping $N_{\mathrm{ch}}$ frequency bands. Moreover, each subchannel is assigned to at most one PUE, which transmits its own data (when available) with fixed power $P_{\mathrm{T}}$ over the assigned subchannel. The status of the $i$ th $\left(i \in\left\{1, \ldots, N_{\mathrm{ch}}\right\}\right)$ subchannel $S_{i}$ is assumed binary, namely:

$$
S_{i}= \begin{cases}S_{0} & \text { with probability } P\left(S_{0}\right) \\ S_{1} & \text { with probability } P\left(S_{1}\right)=1-P\left(S_{0}\right) .\end{cases}
$$

Data transmissions follow a classical model for cellular environments, where the path loss is thoroughly characterized by two parameters: (1) the distance attenuation factor $\alpha$ (adimensional, in the range 2-4) and (2) the standard deviation $\sigma$ (in decibels) of the log-normal shadowing [10].

Each CUE scans all $N_{\text {ch }}$ channels to detect the presence of a primary signal transmission. In other words, the CUEs perform a binary hypothesis test on the presence of primary signals in each

\footnotetext{
* This is a slight abuse of notation, as CUE and PUE are notations typically adopted in cellular systems. However, as smartphones are today advanced sensing systems, we keep this notation also in the realm of WSNs.
} 


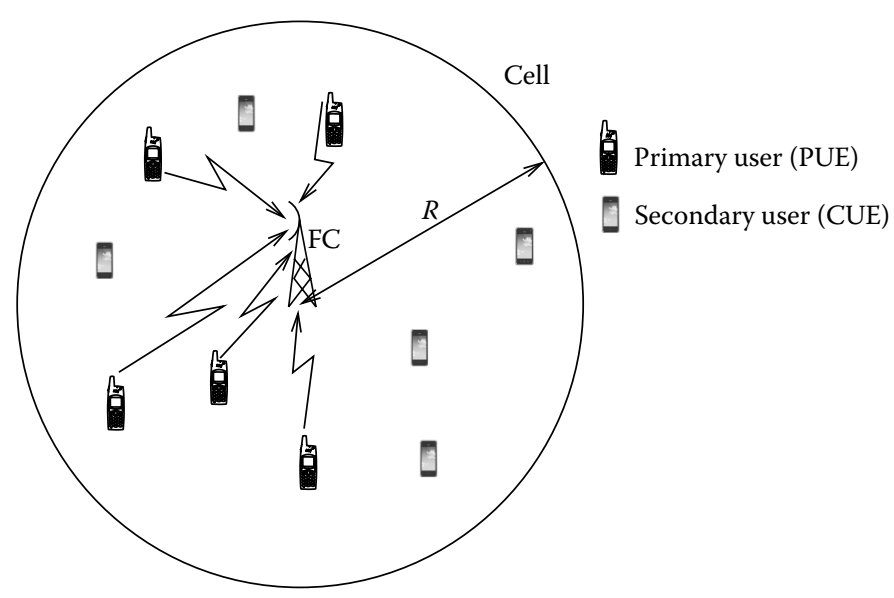

FIGURE 20.1 Cognitive scenario of interest.

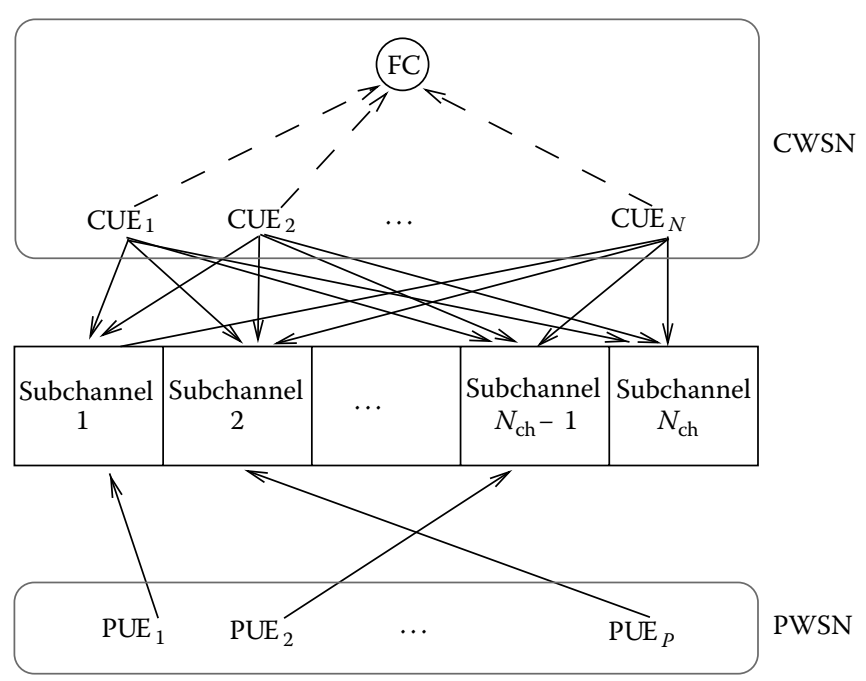

FIGURE 20.2 Logical description of the scenario of interest.

subchannel: A subchannel is idle under hypothesis $S_{0}$ and busy under hypothesis $S_{1}$. As for the signal model, we assume that the primary signal can be modeled as a zero-mean stationary white Gaussian process: This is a reasonable assumption when the CWSN has no a priori knowledge about the possible modulation and pulse shaping formats adopted by the PWSN.

On the basis of the preceding model assumptions, the $k$ th CUE $(k=1, \ldots, N)$ has to distinguish, for the $i$ th subchannel $\left(i=1, \ldots, N_{\mathrm{ch}}\right)$, between two independent Gaussian sequences:

$$
v_{k, i}(\ell)=\left\{\begin{array}{ll}
n_{k, i}(\ell) & \text { if } S_{0} \\
s_{k, i}(\ell)+n_{k, i}(\ell) & \text { if } S_{1}
\end{array} \quad \ell=1, \ldots, m\right.
$$

where $m$ is the number of observed samples-for simplicity, the time index $\ell$ refers to a single block of observations. Note that an implicit assumption in Equation 20.1 is that the phenomenon status ( $S_{0}$ or $S_{1}$ ) does not change over $m$ consecutive observations: This is realistic given that $m$ is typically much shorter than the duration of a primary signal transmission. Moreover, the fact that the $k$ th 
node takes $m$ consecutive observations $\left\{v_{k, i}(\ell)\right\}_{\ell=1}^{m}$ on the $i$ th subchannel guarantees a sort of time diversity in the sensing operation. In Equation 20.1, $s_{k, i}(\ell)$ is the received signal by the $k$ th CUE on the $i$ th subchannel, which is a sequence of i.i.d. zero-mean complex Gaussian random variables with variance $P_{\mathrm{R}}^{(k, i)}$, which corresponds to the received power. The power $P_{\mathrm{R}}^{(k, i)}$ depends on the transmitted power $P_{\mathrm{T}}$ and on the path loss and shadowing terms of each CUE-PUE pair. Note that it is reasonable to assume that the path loss and shadowing terms are constant over all $m$ acquisitions. The noise terms $\left\{n_{k, i}(\ell)\right\}$ are also modeled as i.i.d. zero-mean complex Gaussian random variables with fixed variance $P_{\mathrm{N}}$, constant for all CUEs and subchannels.

Under the observation model (Equation 20.1), an energy detection (ED) scheme is the optimal detector in the Neyman-Pearson sense [11]. In particular, the following decision variable has to be evaluated:

$$
W_{k, i}=\sum_{\ell=1}^{m}\left|v_{k, i}(\ell)\right|^{2}
$$

and the binary decision of the CUE is given by

$$
x_{k, i}= \begin{cases}0 & \text { if } W_{k, i}<\tau \\ 1 & \text { if } W_{k, i} \geq \tau\end{cases}
$$

where $\tau$ is a proper decision threshold. In other words, each CUE decides for 0 if the channel is sensed idle, whereas it decides for 1 if the channel is sensed busy. The local FA and MD probabilities, under the proposed ED scheme, can be defined as follows:

$$
\begin{aligned}
& P_{\mathrm{FA}}^{(k, i)} \triangleq P\left(x_{k, i}=1 \mid S_{0}\right) \\
& P_{\mathrm{MD}}^{(k, i)} \triangleq P\left(x_{k, i}=0 \mid S_{1}\right) .
\end{aligned}
$$

Consequently, the correct detection (CD) probability is

$$
P_{\mathrm{CD}}^{(k, i)}=1-P_{\mathrm{MD}}^{(k, i)}
$$

The $k$ th CUE then generates the decision vector $\boldsymbol{x}_{k}=\left(x_{k, 1}, \ldots, x_{k, N_{\mathrm{ch}}}\right)(k=1, \ldots, N)$, where $x_{k, i} \in$ $\{0,1\}\left(i=1, \ldots, N_{\mathrm{ch}}\right)$ corresponds to its local decision on the absence (0) or presence (1) of a primary signal in the $i$ th subchannel. This vector is then transmitted to the secondary FC, which, on receiving decision vectors from all nodes of the CWSN, applies a proper fusion strategy to make a final decision on the status (free or busy) of each subchannel. The FC can thus broadcast this information to all secondary nodes, possibly with the assignment of the free subchannels to a subset of them, to avoid multiple access interference.

\subsection{SINGLE-NODE PERSPECTIVE}

We now analyze the performance from a single-node perspective. As the subchannels are independent, without loss of generality we focus on a single subchannel and derive the corresponding FA and MD probabilities - for notational simplicity, we drop the superscript/subscript referring to the subchannel. Because each subchannel is assigned to at most one PUE, without loss of generality we also focus on a single PUE. 
With reference to Figure 20.1, assume that CUEs and PUEs are uniformly distributed within the cell with radius $R$ and that the FC is positioned at the cell center. Denote as $X_{k}=x_{k} e^{\jmath 2 \pi \theta_{k}}$ and $X_{\mathrm{p}}=y e^{j 2 \pi \phi}$ the positions of the $k$ th CUE and the PUE, respectively, where $0 \leq x_{k}, y \leq R$ and $0 \leq \theta_{k}$, $\phi \leq 2 \pi$. The distance $d$ between the two nodes is

$$
d_{k}=\left|X_{\mathrm{p}}-X_{k}\right|=\sqrt{x_{k}^{2}+y^{2}-2 x_{k} y \cos \left(\theta_{k}-\phi\right)}
$$

Assuming a fixed transmit power $P_{\mathrm{T}}$ for primary nodes, the power $P_{\mathrm{R}}^{(k)}$ received by the CUEs can be expressed as

$$
P_{\mathrm{R}}^{(k)}=\frac{K}{d_{k}^{\alpha}} h_{k} P_{\mathrm{T}}
$$

where $K$ is the gain at 1 meter from the emitter; $h_{k}$ is the log-normal shadowing coefficient of the link between the PUE and the $k$ th CUE; and $d_{k}$ is the distance between the PUE and the $k$ th CUE. Therefore, the sensing signal-to-noise ratio (SNR) experienced by the $k$ th CUE, with respect to the PUE, can be expressed as follows:

$$
\gamma_{k}\left(d_{k}, h\right)=\frac{P_{\mathrm{R}}^{(k)}}{P_{\mathrm{N}}}=\frac{K h_{k} P_{\mathrm{T}}}{P_{\mathrm{N}} d_{k}^{\alpha}}
$$

The local FA and MD probabilities for ED can then be evaluated as [11]

$$
\begin{aligned}
& P_{\mathrm{FA}}^{(k)}=\Gamma_{\mathrm{u}}\left(m \tau_{\mathrm{N}}, m\right) \\
& P_{\mathrm{MD}}^{(k)}=1-\Gamma_{\mathrm{u}}\left(\frac{m \tau_{\mathrm{N}}}{1+\gamma_{k}\left(d_{k}, h\right)}, m\right)
\end{aligned}
$$

where $\tau_{\mathrm{N}}=P_{\mathrm{N}} \tau$ is the normalized threshold (with $\tau$ introduced in Equation 20.2) and $\Gamma_{\mathrm{u}}$ is the upper incomplete gamma function:

$$
\Gamma_{\mathrm{u}}(a, n) \triangleq \int_{a}^{\infty} x^{n-1} e^{-x} \mathrm{~d} x
$$

Note that the FA probability is the same for all CUEs and does not depend on their distances from the PUE, that is, $P_{\mathrm{FA}}^{(k)}=P_{\mathrm{FA}}$. The MD probability, instead, depends on the distance $d_{k}$ and on the shadowing term $h_{k}$. Averaging with respect to the statistical distribution of the shadowing term, the following expression for the average MD probability at distance $d_{k}$ is obtained:

$$
\bar{P}_{\mathrm{MD}}^{(k)}=1-\frac{1}{\sqrt{2 \pi \sigma^{2}}} \int_{-\infty}^{\infty} \Gamma_{\mathrm{u}}\left(\frac{m \tau_{\mathrm{N}}}{1+\gamma_{k}\left(d_{k}, 10^{S / 10}\right)}, m\right) e^{-\frac{S^{2}}{2 \sigma^{2}}} \mathrm{~d} S .
$$

Even though the integral in Equation 20.3 has no closed-form solution, it can be numerically evaluated. Finally, the average MD probability for any CUE, denoted as $\bar{P}_{\mathrm{MD}}$, can be obtained from Equation 20.3 by averaging over the distance $d_{k}$, thus obtaining:

$$
\bar{P}_{\mathrm{MD}}=\int_{\rho} \bar{P}_{\mathrm{MD}}^{(k)}(\rho) f_{D}(\rho) \mathrm{d} \rho
$$


where $f_{D}(\rho)$ is the probability density function (PDF) of the CUE-PUE distance. The average CD probability for CUE, denoted as $\bar{P}_{\mathrm{CD}}$, can be straightforwardly expressed as

$$
\bar{P}_{\mathrm{CD}}=1-\bar{P}_{\mathrm{MD}}
$$

Expression 20.4 for $\bar{P}_{\mathrm{MD}}$ is an average measure for all possible CUEs. The $\operatorname{PDF} f_{D}(\rho)$ is associated with the distance between two randomly chosen points in a circle and, according to the Crofton fixed points theorem [12], can be given by the following expression:

$$
f_{D}(\rho)=2 \frac{\rho}{R^{2}}\left[\frac{2}{\pi} \arccos \left(\frac{\rho}{2 R}\right)-\frac{\rho}{\pi R} \sqrt{1-\frac{\rho^{2}}{4 R^{2}}}\right] \quad 0 \leq \rho \leq 2 R .
$$

The choice of a proper value for the threshold $\tau_{\mathrm{N}}$ is crucial to maximize the ultimate system performance. Indeed, a small value of $\tau_{\mathrm{N}}$ yields frequent FAs, whereas a high value of $\tau_{\mathrm{N}}$ entails a high MD probability. The optimized value of $\tau_{\mathrm{N}}$ obviously depends on the sensing SNR experienced by each CUE, which, in turn, depends on several other uncontrollable characteristics, such as the positions of CUEs/PUEs and shadowing. We preliminarily observe that the selection of a different and optimized threshold for each CUE would involve a huge amount of message exchange between the FC and the CUEs. Therefore, we make the reasonable assumption that the threshold is a predefined system parameter to be optimized off-line (e.g., in a training phase) through statistical considerations. More generally, we make the assumption that the FC has no knowledge about the actual positions of CUEs and PUEs inside the cell.

\subsection{NETWORK PERSPECTIVE}

We now analyze the performance from a network perspective, considering the use of the local decisions coming from all the CUEs, considering possible data fusion rules. In particular, in Section 20.4.1 we first focus on an uncoded scenario with error-free communication links. Then, in Section 20.4.2 we extend our analysis to the presence of communication noise and channel coding.

\subsubsection{Uncoded Scenario with No Communication Noise}

We first consider the case where data (i.e., local decisions on the statuses of the subchannels) are transmitted as uncoded by each CUE to the secondary FC, using a set of orthogonal error-free communication channels. This makes it possible to derive the ultimate performance limits that can be achieved in this scenario. In reality, noisy communications from CUEs to the secondary FC are likely to degrade the system performance, as shown by means of simulations in Section 20.5.

To derive the network-wide performance in the absence of channel coding, we now consider all the $N_{\mathrm{ch}}$ subchannels, where the characteristics of the local decisions by the CUEs, in terms of FA and MD probabilities, have been derived in Section 20.3. Using the considered observation model, it is possible to write the a priori joint (among all CUEs) probability mass function (PMF) of the decisions for the $i$-th subchannel $\left(i \in\left\{1, \ldots, N_{\mathrm{ch}}\right\}\right)$ as [13]

$$
\begin{aligned}
P\left(\boldsymbol{x}_{i}\right) & =P\left(\boldsymbol{x}_{i} \mid S_{i}=S_{0}\right) P\left(S_{0}\right)+P\left(\boldsymbol{x}_{i} \mid S_{i}=S_{1}\right) P\left(S_{1}\right) \\
& =P\left(S_{0}\right) \prod_{k \in \mathcal{X}_{0}}\left(1-P_{\mathrm{FA}}\right)^{k} \prod_{k \in \mathcal{X}_{1}}\left(P_{\mathrm{FA}}\right)^{N-k}+P\left(S_{1}\right) \prod_{k \in \mathcal{X}_{0}}\left(1-\bar{P}_{\mathrm{CD}}\right)^{k} \prod_{k \in \mathcal{X}_{1}}\left(\bar{P}_{\mathrm{CD}}\right)^{N-k}
\end{aligned}
$$


where

$$
\begin{aligned}
& \mathcal{X}_{0}=\left\{h \in\{1, \ldots, N\}: x_{k, h}=0\right\} \\
& \mathcal{X}_{1}=\left\{j \in\{1, \ldots, N\}: x_{k, j}=1\right\}
\end{aligned}
$$

Obviously, $\mathcal{X}_{0} \cup \mathcal{X}_{1}=\{1, \ldots, N\}$.

As the communication channels between the CUEs and the secondary FC are error free, the transmitted data are received correctly and the fusion rule at the FC can be written as

$$
\Lambda_{i}=\sum_{k=1}^{N} x_{k, i} \underset{S_{0}}{\stackrel{S_{1}}{\gtrless}} \boldsymbol{T}
$$

where $T$ is a "global" decision threshold (i.e., equal for all subchannels) to be optimized. In other words, the hypothesis testing problem turns out be a counting problem: the number of local decisions, at the CUEs, in favor of $S_{1}$ is first counted and then compared with the threshold $T$. The fusion rule (Equation 20.6) is shown to be optimal under the assumption, as in this chapter, of blind detection, that is, the FC has no knowledge about the actual sensing accuracy (in terms of local FA and MD probabilities) of each CUE [14].

Let us now evaluate the performance at the FC by computing the final CD and FA probabilities, denoted as $P_{\mathrm{CD}, \mathrm{f}}$ and $P_{\mathrm{FA}, \mathrm{f}}$, respectively - note that these probabilities do not depend on the subchannel. To this end, we approximate the performance of the system following an approach similar to that in Ref. [14]. In particular, we assume that all users are characterized by the same $\bar{P}_{\mathrm{MD}}$ given by Equation 20.4, regardless of the position of the PUE. However, unlike Ref. [14], we do not consider a Gaussian approximation for $\Lambda$, as the number of CUEs may not necessarily be large. As the observations $\left\{x_{k}\right\}$ are i.i.d. with a Bernoulli distribution, one can write

$$
\begin{aligned}
P_{\mathrm{CD}, \mathrm{f}} & =P\left(\Lambda>T \mid S_{1}\right) \\
& \simeq \sum_{k=T}^{N}\left(\begin{array}{l}
N \\
k
\end{array}\right)\left(\bar{P}_{\mathrm{CD}}\right)^{k}\left(1-\bar{P}_{\mathrm{CD}}\right)^{N-k} .
\end{aligned}
$$

As the local FA probability does not depend on the position of the PUE, the FA probability at the FC has exactly the following expression:

$$
\begin{aligned}
P_{\mathrm{FA}, \mathrm{f}} & =P\left(\Lambda>T \mid S_{0}\right) \\
& =\sum_{k=T}^{N}\left(\begin{array}{l}
N \\
k
\end{array}\right)\left(P_{\mathrm{FA}}\right)^{k}\left(1-P_{\mathrm{FA}}\right)^{N-k} .
\end{aligned}
$$

At this point, one can observe that the probabilities in Equations 20.7 and 20.8 depend on the local and global thresholds, that is, one can write:

$$
\begin{aligned}
& P_{\mathrm{CD}, \mathrm{f}}=\mathcal{G}\left(\tau_{\mathrm{N}}, T\right) \\
& P_{\mathrm{FA}, \mathrm{f}}=\mathcal{F}\left(\tau_{\mathrm{N}}, T\right)
\end{aligned}
$$


where $\mathcal{G}(\cdot, \cdot)$ and $\mathcal{F}(\cdot, \cdot)$ are proper functions. The optimized values of the local $\left(\tau_{\mathrm{N}}\right)$ and global $(T)$ thresholds can be determined by observing that protecting primary users against secondary interference is, in general, more important than giving opportunities to secondary users. Therefore, we consider a Neyman-Pearson detector at the FC where we fix the requested CD probability, that is, we impose $P_{\mathrm{CD}, \mathrm{f}}=P_{\mathrm{CD}, \mathrm{tg}}$. For each possible value of $\tau_{\mathrm{N}}$, the optimal value of the threshold $T$, denoted as $T^{*}$, can be determined by finding the maximum value (if existing) of $T$ that allows to achieve $P_{\mathrm{CD}, \mathrm{tg}}$, that is,

$$
T^{*}\left(\tau_{\mathrm{N}}\right)=\max \left\{T \in \mathbb{N}: \mathcal{G}\left(\tau_{\mathrm{N}}, T\right)=P_{\mathrm{CD}, \mathrm{gt}}\right\}
$$

In Equation 20.9, the maximum value is considered because $\mathcal{G}\left(\tau_{\mathrm{N}}, T\right)$ is, as shown later, a decreasing function of $T$ for fixed $\tau_{\mathrm{N}}$. The optimal value of $\tau_{\mathrm{N}}$, denoted as $\tau_{\mathrm{N}}^{*}$, is then selected as the value that allows to achieve the minimum $P_{\mathrm{FA}, \mathrm{f}}$ :

$$
\tau_{\mathrm{N}}^{*}=\underset{\tau_{\mathrm{N}}}{\arg \min } \mathcal{F}\left[\tau_{\mathrm{N}}, T^{*}\left(\tau_{\mathrm{N}}\right)\right]
$$

We now show an illustrative example of the general scenario of interest shown in Figure 20.1. In particular, the main system parameters are the following: $R=1 \mathrm{~km}, P_{\mathrm{T}}=30 \mathrm{~mW}, \alpha=4, \sigma=5 \mathrm{~dB}$, $P_{\mathrm{N}}=-110 \mathrm{dBm}$, and $m=10$. In Figure 20.3, $P_{\mathrm{CD}, \mathrm{f}}$ is shown, as a function of $T$, for $N=15$ and various values of $\tau_{\mathrm{N}}$. In all cases, the target CD probability $P_{\mathrm{CD} \text {,tgt }}$ is set to 0.95 . One can observe that the optimal value of $T$ depends on $\tau_{\mathrm{N}}$, for instance, for $\tau_{\mathrm{N}}=1$ the optimal threshold is $T=6$, whereas $T=$ 1 for $\tau_{\mathrm{N}}=1.6$. These values are then used to determine the optimal values of $\tau_{\mathrm{N}}$, as shown in Figure 20.4, where $P_{\mathrm{FA}, \mathrm{f}}$ is shown, as a function of $\tau_{\mathrm{N}}$, for various values of $T$. The points highlighted with circles correspond to those obtained from Figure 20.3. Because the optimized thresholds correspond to the minimum FA, in the considered settings the optimized thresholds are $\left(T^{*}, \tau_{\mathrm{N}}^{*}\right)=(1,1.8)$.

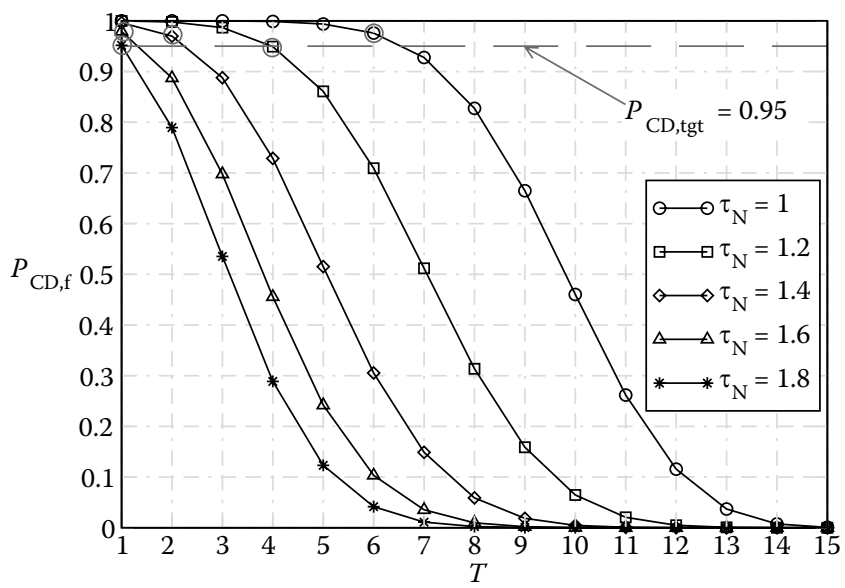

FIGURE 20.3 $P_{\mathrm{CD}, \mathrm{f}}$ as a function of $T$, for $N=15$ and various values of $\tau_{\mathrm{N}}$. In all cases, the target CD probability $P_{\mathrm{CD}, \mathrm{tgt}}$ is set to 0.95 . 


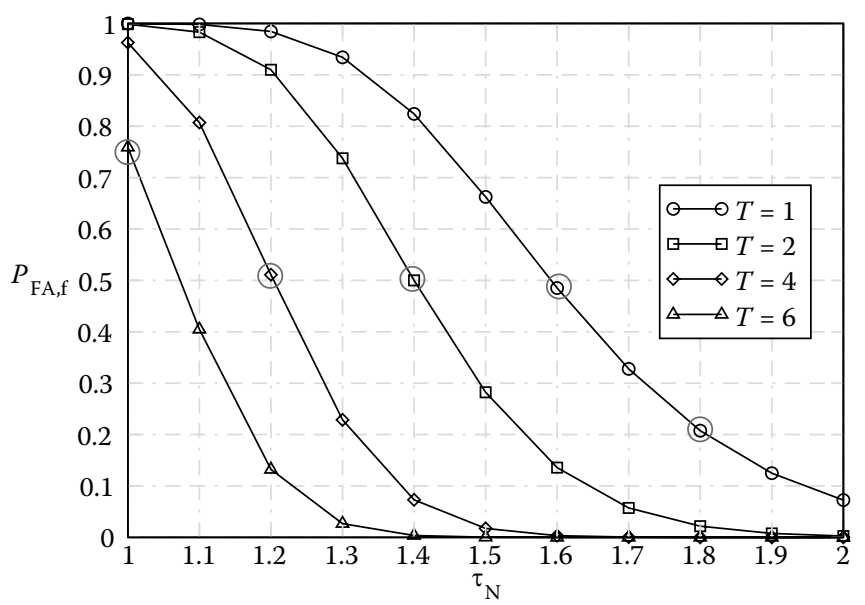

FIGURE 20.4 $P_{\text {FA, f }}$, as a function of $\tau_{\mathrm{N}}$, for various values of $T$. The points highlighted with circles correspond to those obtained from Figure 20.3.

\subsubsection{Coded Scenario with Communication Noise}

Consider now a scenario where the data transmitted by the CUEs to the FC are corrupted by communication noise. For simplicity, assume that the communication links are orthogonal and affected by additive white Gaussian noise (AWGN), so that the observable model at the FC is

$$
r_{k, i}=\tilde{x}_{k, i}+w_{k, i} \quad k=1, \ldots, N \quad i=1, \ldots, N_{\mathrm{ch}}
$$

where $\left\{\tilde{x}_{k, i}\right\}_{i=1}^{N_{\text {ch }}}$ is the binary data sequence obtained by channel encoding (with coding rate $r_{\text {cod }}$ ) the decision sequence $\left\{x_{k, i}\right\}_{i=1}^{N_{\text {ch }}}$. The communication channel is characterized by an SNR denoted as $\gamma_{\mathrm{ch}}^{(k)} *$ In Section 20.5, a common average value of the communication SNR, denoted as $\gamma_{\mathrm{ch}}$, will be considered. The choice of proper channel coding strategies is an interesting open issue, due to the fact that the decision packet length is typically small (in fact, it corresponds to the number $N_{\text {ch }}$ of subchannels). However, this problem goes beyond the scope of this chapter-in Section 20.5, a regular low-density parity-check (LDPC) code will be considered.

Because the transmitted data are inherently correlated according to the PMF in Equation 20.5, inspired by the work in Ref. [9], we use JCD at the receiver to improve the detection/decoding performance. In this case, $N$ subdecoders, one per CUE, are present at the AP. Each subdecoder works on the basis of its channel LLRs and the a priori soft information obtained from the soft-output information generated by the other subdecoders (associated with the remaining CUEs), properly combined taking into account the source correlation. The corresponding scheme of the JCD receiver is shown in Figure 20.5. This procedure is then iterated to refine at each step the decisions of each decoder.

The log-likelihood ratio (LLR) relative to the $i$ th observable at the input of the $k$ th subdecoder can be written as follows [15]:

$$
\mathcal{L}_{i, \mathrm{in}}^{(k)}= \begin{cases}\mathcal{L}_{i, \mathrm{ch}}^{(k)}+\mathcal{L}_{i, \mathrm{ap}}^{(k)} & i=1, \ldots, N_{\mathrm{ch}} \\ \mathcal{L}_{i, \mathrm{ch}}^{(k)} & i=N_{\mathrm{ch}}+1, \ldots, N_{\mathrm{ch}} / r_{\mathrm{cod}}\end{cases}
$$

\footnotetext{
* We are making the reasonable assumption that the SNR is constant over the transmission of an entire packet $\boldsymbol{x}_{k}=\left(x_{k, 1}, \ldots, x_{k, N_{\mathrm{ch}}}\right)$ and changes independently from CUE to CUE.
} 


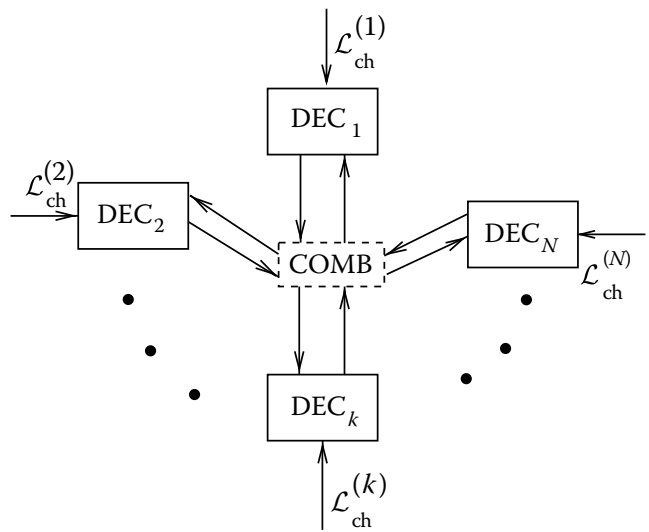

FIGURE 20.5 JCD algorithm and iterative decoding.

where the channel LLR can be written as

$$
\mathcal{L}_{i, \mathrm{ch}}^{(k)}=\frac{2 r_{i, k}}{\sigma_{\mathrm{N}}^{2}} \quad k=1, \ldots, N
$$

with $\sigma_{\mathrm{N}}^{2}$ being the variance of the AWGN. The a priori component of the LLR can be instead written as [9]

$$
P\left(x_{i, k}\right)=\sum_{x_{i}^{\prime}} \underbrace{P\left(x_{i, k}=0 \mid x_{i}^{\prime}\right)}_{\text {a priori source correl. }} \prod_{\substack{\ell=1 \\ \ell \neq k}}^{N} \underbrace{\hat{P}\left(x_{i, \ell}\right)}_{\text {from decoder } \ell} .
$$

Because each decoder outputs the LLRs on the bits of the information sequence, the following soft-input fusion (SF) rule can be used:

$$
\hat{S}_{i}=\underset{S_{i}=0,1}{\operatorname{argmax}} \beta_{i} \sum_{\left\{\boldsymbol{x}_{i}\right\}} \underbrace{\frac{P\left(\boldsymbol{x}_{i} \mid S_{i}\right) P\left(S_{i}\right)}{\sum_{S^{*}=0,1} P\left(\boldsymbol{x}_{i} \mid S^{*}\right) P\left(S^{*}\right)}}_{\text {from the correlation model }} \underbrace{\prod_{j=1}^{n} P\left(x_{i, j} \mid \mathcal{L}_{i}^{(j)}\right)}_{\text {from decoder LLRs }}
$$

where

$$
\beta_{i} \triangleq \beta\left(1-S_{i}\right)+(1-\beta) S_{i} \quad i \in\{0,1\}
$$

with $\beta \in(0,1)$. In other words, the fusion rule (Equation 20.11), derived in Ref. [9] from a maximum a posteriori probability (MAP) criterion, can still be used in this scenario using proper coefficients $\beta_{0}=$ $\beta$ and $\beta_{1}=1-\beta$ to obtain proper FA and CD probabilities. These coefficients need to be optimized according to a given criterion: In particular, in this chapter we consider the same procedure used in Section 20.4.1 to optimize $T$. In other words, for each value of $\tau_{\mathrm{N}}$, the optimal value of the threshold $\beta$, denoted as $\beta^{*}$, can be determined by finding the maximum value (if it exists) of $\beta$ that allows to achieve $P_{\mathrm{CD}, \mathrm{tgt}}$. The optimal value of $\tau_{\mathrm{N}}$, denoted as $\tau_{\mathrm{N}}^{*}$, is then selected as the value which allows to achieve, for $\beta=\beta^{*}$, the minimum $P_{\mathrm{FA}, \mathrm{f}}$. Note also that these optimal values obviously depend on the sensing SNR. 


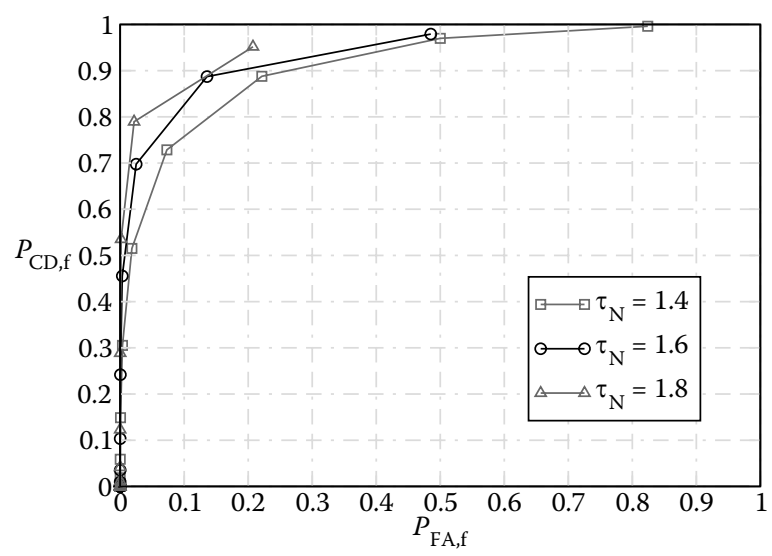

FIGURE 20.6 Uncoded ROC for $N=15$ and various values of $\tau_{\mathrm{N}}$.

Moreover, a hard-input fusion (HF) rule can be considered if the LLRs at the output of the channel decoders are quantized, thus obtaining an estimate of the local decisions $\hat{\boldsymbol{x}}_{i}\left(i=1, \ldots, N_{\mathrm{ch}}\right)$. From a fusion point of view, the estimated decisions $\left\{\hat{\boldsymbol{x}}_{i}\right\}_{i=1}^{N_{\mathrm{ch}}}$ are uncoded and, therefore, the same fusion rule as in Equation 20.6 can be applied:

$$
\Lambda_{i, \mathrm{cod}}=\sum_{k=1}^{N} \hat{x}_{k, i} \stackrel{S_{1}}{\gtrless} T_{S_{0}}
$$

where $T_{\text {cod }}$ is a proper global threshold. The same optimization procedure for local $\tau_{\mathrm{N}}$ and global $T_{\text {cod }}$ thresholds described at the end of Section 20.4.1 can be applied in this case as well.

\subsection{NUMERICAL RESULTS}

In this section, we present simulation results considering the same scenario as in Section 20.4.1. We assume that the frequency band is divided into $N_{\mathrm{ch}}=100$ subchannels and, in the presence of channel coding, a half-rate (i.e., $\left.r_{\text {cod }}=1 / 2\right)(3,6)$ regular LDPC code, with pseudo-random parity-check matrix generation, is considered. The JCD algorithm summarized in Section 20.4.2 is performed with five external iterations, while each LDPC decoder performs internal iterations until a valid codeword is obtained or a maximum number of 50 internal iterations is reached. To eliminate statistical fluctuations of the communication noise, the results in the presence of channel coding are averaged over 100 different trials. In both uncoded and coded scenarios, results are presented in terms of the receiver operating characteristic (ROC) curve, for the binary hypothesis testing problem of interest, showing the CD probability as a function of the FA probability [16].

\subsubsection{UnCODED SCENARIO}

In Figure 20.6, the uncoded ROC is shown for $N=15$ and various values of $\tau_{\mathrm{N}}$. Each point of the curves corresponds to a different value of $T \in\{1,2, \ldots, N\}$; in particular, $T=1$ corresponds to the point in the upper right corner of the figure.* Note that for increasing values of $T$, a smaller FA probability can be obtained at the price of a lower CD probability. Moreover, increasing $\tau_{\mathrm{N}}$ for a

\footnotetext{
* Simulation results for the uncoded case, not shown here for brevity, are in agreement with the theoretical results obtained from our analytical framework in Section 20.4.1.
} 


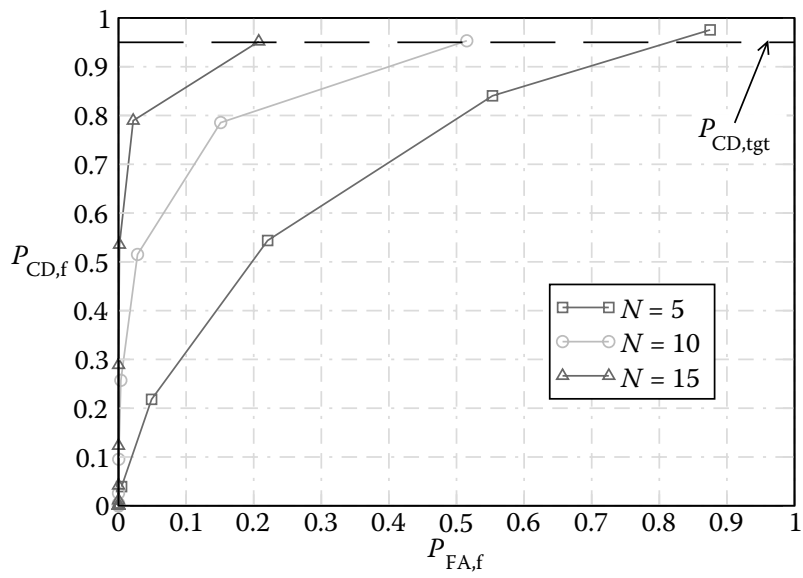

FIGURE 20.7 Uncoded ROC for various values of $N$, considering the optimal values of $T$ and $\tau_{\mathrm{N}}$ obtained with $P_{\mathrm{CD}, \mathrm{tgt}}=0.95$.

fixed value of $T$ allows to reduce both FA and CD probabilities, thus showing the inherent tradeoff between these two quantities.

In Figure 20.7, the uncoded ROC is shown for various values of $N$, considering the optimal values of $T$ and $\tau_{\mathrm{N}}$ obtained with $P_{\mathrm{CD}, \mathrm{tgt}}=0.95$. In particular, for $N=5$ the optimal point is $\left(T^{*}=1, \tau_{\mathrm{N}}=1.1\right)$, for $N=10\left(T^{*}=1, \tau_{\mathrm{N}}=1.5\right)$, and for $N=15\left(T^{*}=1, \tau_{\mathrm{N}}=1.8\right)$, as detailed in Table 20.1. Note that in all presented cases $T=1$, that is, the optimal decision rule is the OR decision rule. This means that, if CUE observations are available without errors are the FC, it is sufficient to have at least one of them in favor of $S_{1}$ to decide for this status. Moreover, for increasing values of $N$ both $\tau_{\mathrm{N}}$ and $T$ increase as well and the minimum possible $P_{\mathrm{FA}, \mathrm{f}}$ can be noticeably reduced from $0.88(N=5)$ to $0.52(N=10)$ and $0.21(N=15)$. Obviously, these results depend on the considered target CD probability, as summarized in Table 20.1. In particular, one can observe that with $N=10$ and $P_{\mathrm{CD}, \mathrm{tgt}}$ the minimum FA probability is achieved by fusing two decisions instead of one.

\subsubsection{Coded SCEnARio}

In Figure 20.8, we show the ROC curves obtained considering $N=10, P_{\mathrm{CD} \text {,tgt }}=0.9$, and different values of $\gamma_{\mathrm{ch}}$. The performance of SF is compared with that of HF. One can observe that the better the communication channels (i.e., the higher the channel SNR), the closer the performance to the theoretical limit given by the uncoded system: in particular, the performance with $\gamma_{\mathrm{ch}}=10 \mathrm{~dB}$ overlaps with the theoretical limit given by the uncoded system. Moreover, in the presence of a bad communication link quality, the use of SF allows one to obtain better performance than HF because the likelihood information coming from the decoder is properly exploited. The ROC curves are

TABLE 20.1

Optimal Working Points for the Uncoded Case and Various Values of $N$ and $\boldsymbol{P}_{\mathrm{CD}, \mathrm{tg}}$

$\begin{array}{lccc}\boldsymbol{P}_{\mathrm{CD}, \text { tgt }} & \boldsymbol{N}=\mathbf{5} & \mathbf{N}=\mathbf{1 0} & \boldsymbol{N}=\mathbf{1 5} \\ 0.9 & T^{*}=1, \tau_{\mathrm{N}}^{*}=1.2 & T^{*}=1, \tau_{\mathrm{N}}^{*}=1.6 & T^{*}=1, \tau_{\mathrm{N}}^{*}=2.1 \\ 0.95 & T^{*}=1, \tau_{\mathrm{N}}^{*}=1.1 & T^{*}=1, \tau_{\mathrm{N}}^{*}=1.5 & T^{*}=1, \tau_{\mathrm{N}}^{*}=1.8 \\ 0.99 & T^{*}=1, \tau_{\mathrm{N}}^{*}=1 & T^{*}=2, \tau_{\mathrm{N}}^{*}=1.1 & T^{*}=1, \tau_{\mathrm{N}}^{*}=1.4\end{array}$




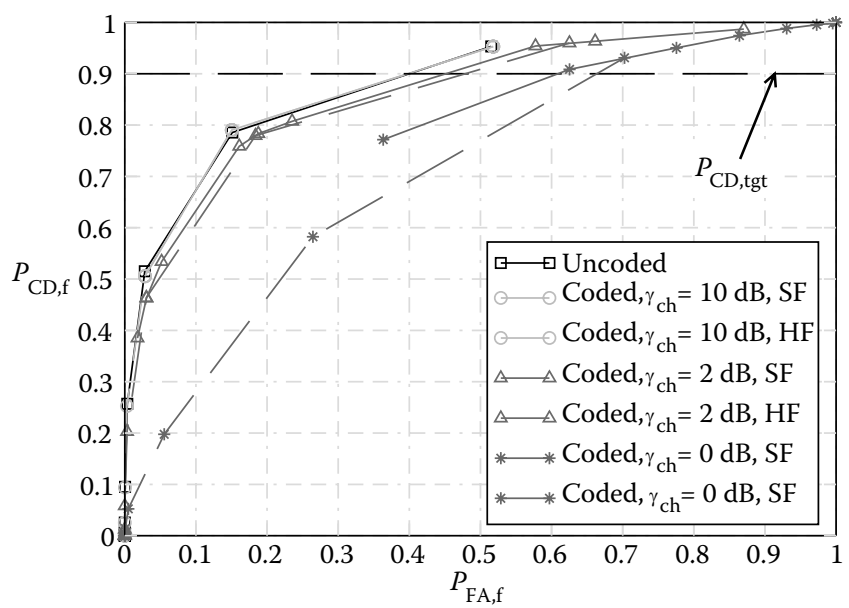

FIGURE 20.8 ROC curves for $N=10, P_{\mathrm{CD}, \mathrm{tgt}}=0.9$, and different values of $\gamma_{\mathrm{ch}}$. The performance of SF is compared with that of HF.

shown for the optimal values of thresholds, for example, $T^{*}$ and $\tau_{\mathrm{N}}^{*}$ in the uncoded case (indicated as reference in Figure 20.8), $\beta^{*}$ and $\tau_{\mathrm{N}}^{*}$ for the coded case and SF, and $T_{\text {cod }}^{*}$ and $\tau_{\mathrm{N}}^{*}$ for the coded case and HF. In particular, for high SNR (i.e., $\gamma_{\mathrm{ch}}=10 \mathrm{~dB}$ ) the optimal value is $\tau_{\mathrm{N}}^{*}=1.6$ for both uncoded and coded (either HF or SF) cases. When the SNR decreases, for example, $\gamma_{\mathrm{ch}}=2 \mathrm{~dB}$, the optimal value is, in the coded case (either HF or SF), $\tau_{\mathrm{N}}^{*}=1.7$. Finally, for very bad communication link quality, for example, $\gamma_{\mathrm{ch}}=0 \mathrm{~dB}, \tau_{\mathrm{N}}^{*}=1.3$ with $\mathrm{HF}$ and 2 with SF. The fact that the optimized threshold differs in the coded case for at least medium-low communication SNRs, leads to the conjecture that the choice of $\tau_{\mathrm{N}}$ changes the correlation model, which should be properly taken into account. Therefore, the dimensionality of the optimization problem increases. This issue will be the subject of our future work.

\subsection{CONCLUDING REMARKS}

In this chapter, we have analyzed a cognitive WSN, where CUEs sense the frequency spectrum and cooperate to estimate the free subchannels that can be used to transmit their data. First, a simple analytical framework for characterizing the local sensing performance per subchannel, in terms of MD and FA probabilities, has been derived for a scenario with uncoded transmissions over errorfree communication channels. This allows one to determine the ultimate achievable performance in such a scenario. Then, we have considered a realistic scenario with communication noise and the use of channel coding. In this context, the performance of JSCC schemes with JCD at the receiver has been investigated. Our results have shown the beneficial impact of JCD at the receiver (with improved quality of the subchannel status estimation), thus making it possible to achieve performance close to the theoretical limit for sufficiently large values of the SNR.

\section{REFERENCES}

1. D. Datla, A. Wyglinski, and G. Minden. A spectrum surveying framework for dynamic spectrum access networks. IEEE Transactions on Vehicular Technology, 58(2):4158-4168, 2009.

2. J. Mitola, and G. Q. Maguire. Cognitive radio: Making software radios more personal. IEEE Personal Communications, 6(4):13-18, 1999.

3. J. Mitola. Cognitive Radio: An Integrated Agent Architecture for Software-Defined radio. PhD dissertation, Royal Institute of Technology, Stockholm, 2000. 
4. S. Heykin. Cognitive radio: Brain-empowered wireless communications. IEEE Journal on Selected Areas in Communication, 23:201-220, 2005.

5. S. Heykin. Fundamentals Issues in Cognitive Radio. Springer, New York, 2007.

6. R. Tandra, M. Mishra, and A. Sahai. What is a spectrum hole and what does it take to recognize one? Proceedings of IEEE, 97:824-848, 2009.

7. G. Ganesan, and G. Y. Li. Cooperative spectrum sensing in cognitive radiopart I: Two user networks. IEEE Transactions on Wireless Communications, 6(6):2204-2213, 2007.

8. G. Ganesan, and G. Y. Li. Cooperative spectrum sensing in cognitive radiopart II: Multiuser networks. IEEE Transactions on Wireless Communication, 6(6):2214-2222, 2007.

9. G. Ferrari, M. Martalò, and A. Abrardo. Information fusion in wireless sensor networks with source correlation. Elsevier Information Fusion, 15:80-89, 2014.

10. A. Goldsmith. Wireless Communications. Cambridge University Press, New York, 2005.

11. S. M. Kay. Fundamentals of Statistical Signal Processing: Estimation Theory. Prentice-Hall, Upper Saddle River, NJ, 1993.

12. D. Moltchanov. Distance distributions in random networks. Ad Hoc Networks, 10(6):1146-1166, 2012.

13. A. Papoulis. Probability, Random Variables and Stochastic Processes. McGraw-Hill, New York, 1991.

14. R. Niu, P. K. Varshney, and Q. Cheng. Distributed detection in a large wireless sensor network. Information Fusion, 7(4):380-394, 2006.

15. A. Abrardo, G. Ferrari, M. Martalò, and F. Perna. Feedback power control strategies in wireless sensor networks with joint channel decoding. MDPI Sensors, 9(11):8776-8809, 2009.

16. T. Fawcett. An introduction to ROC analysis. Pattern Recognition, 27(8):861-874, 2006. 